\title{
Guy Bodenmann
}

\section{Positionsbestimmung in der Paar- und Familienpsychologie}

\author{
Current situation of couple and family psychology
}

\begin{abstract}
Zusammenfassung
Dieser Beitrag gibt einen Überblick über gewisse Themen und Strömungen in der Paar- und Familienpsychologie, die aktuell besondere Aufmerksamkeit erfahren. Diese werden nach dem Gesichtspunkt der familienpsychologischen Grundlagenforschung versus der familienpsychologischen Anwendungsforschung unterschieden. Diese $\mathrm{Zu}$ sammenstellung erhebt keinen Anspruch auf Vollständigkeit, sondern zeigt lediglich einige Tendenzen auf. Forschungsthemen, die künftig noch mehr Beachtung finden sollten, werden ebenfalls kurz gestreift.

Schlagworte: Familie, Partnerschaft, Scheidung, Stress, Bindung, Paartherapie.
\end{abstract}

\begin{abstract}
This article tries to resume some of the theoretical and empirical issues that attract currently high attention within the field of marital and family psychology. Although no exhausting overview can be given, some relevant topics are presented and discussed with regard to basic research in family psychology and their application in prevention and psychotherapy. Themes that merit more consideration in the future are also addressed.
\end{abstract}

Key words: family, couple, divorce, stress, attachment, marital therapy.

\section{Einleitung}

Die psychologische Paar- und Familienforschung gehört erst seit den 1980er Jahren zum festen Bestandteil der psychologischen Forschung in den deutschsprachigen Ländern Europas, richtig zu etablieren begann sie sich jedoch erst in den 1990er Jahren, zeitgleich mit dem Erscheinen der wegweisenden Schrift „Familienpsychologie " von Schneewind (1991). Seither hat sich die Paar- und Familienforschung im deutschen Sprachraum markant entfaltet, was sich mitunter in der stetigen Zunahme von Publikationen in diesem Bereich niederschlägt. Schneewind (2000) hat dieser Entwicklung in seinem Buch „Familienpsychologie im Aufwind: Brückenschlag zwischen Forschung und Praxis" ebenso Ausdruck gegeben wie Walper und Pekrun (2001) in „Familie und Entwicklung. Perspektiven der Familienpsychologie“. 
Dieses wachsende Interesse an der Familienforschung spiegelt sich zudem in der Gründung von einschlägigen Instituten seit Mitte der 1990er Jahre wider. So wurden 1994 zeitgleich Familienforschungsinstitute in Deutschland (Staatsinstitut für Familienforschung an der Universität Bamberg), Österreich (Österreichisches Institut für Familienforschung) und der Schweiz (Institut für Familienforschung und -beratung der Universität Fribourg) errichtet, die seither eine rege wissenschaftliche Aktivität entwickelt haben, wie die jährlich erscheinenden Jahresberichte dieser drei Institutionen erkennen lassen. Das steigende Interesse an der psychologischen Familienforschung zeigt auch die Gründung der Academy of Family Psychology im Jahre 1990, bei welcher von Anfang an namhafte deutschsprachige Forscherinnen und Forscher mitwirkten sowie der interdisziplinären European Society on Family Relations im Jahre 2002 oder die alle zwei Jahre stattfindende Münchner Tagung zur Familienpsychologie unter der Leitung von Klaus Schneewind, die dieses Jahr zum vierten Mal einen Überblick über die State of the Art im Bereich der Paar- und Familienforschung in Deutschland, Österreich und der Schweiz gibt. Ein weiteres Beispiel für die heutige feste Verankerung der Familienforschung im öffentlichen Bewusstsein (wobei die psychologische Familienforschung hier eine zentrale Rolle spielt) stellt das Online-Familienhandbuch von Fthenakis und Textor (2001) dar, welches seit seiner Aufschaltung vor rund vier Jahren eine beeindruckende Resonanz erfuhr. Gemäß Angaben von Martin Textor wurde das Online-Familienhandbuch bis im Mai 2005 von 233.000 Benutzern besucht, die 1,2 Millionen Seiten aufriefen.

Diese Beispiele zeigen, dass sich die Paar- und Familienpsychologie im deutschen Sprachraum einen festen Platz in der Forschung, in der Universitätslandschaft und im öffentlichen Bewusstsein geschaffen hat. Im Folgenden soll nun ein Überblick über die Aktivitäten dieses Forschungsbereichs gegeben werden, wobei in Anlehnung an Schneewind (1999) die Positionsbestimmung innerhalb der familienpsychologischen Grundlagenforschung und der familienpsychologischen Anwendungsforschung vorgenommen wird. In der aktuellen Familienpsychologie werden vielfältige Themen bearbeitet (siehe Tabelle 1).

Tabelle 1: Einige aktuelle Themen der Paar- und Familienpsychologie

- Bedeutung verschiedener Familienformen und ihre Lebensbedingungen

- Familien in ökonomischer Deprivation und Armut

- familiale Beziehungsmuster und ihre Entwicklung

- Geschwister- und Zwillingsbeziehungen

- Anpassung der Kinder an neue Familienformen und Scheidung

- Sozialisation im Familienkontext und im sozialen Wandel

- die Rolle der Väter in der Familie und für die Entwicklung der Kinder

- Rollenteilung und moderne Paarbeziehungen

- familiäre Gewalt

- Work-Life-Balance (Verträglichkeit von Familie und Beruf)

Dabei soll insbesondere auf Schwerpunkte und aktuelle Strömungen in diesen beiden Bereichen eingegangen werden, welche in den letzten Jahren besondere Beachtung und Resonanz erfahren haben, was sich zum einen in ihrem Publikationsvolumen niederschlägt (siehe Abbildung 1) wie auch in Überblicksartikeln (vgl. z.B. Gottman/Notarius 2002). 
Abbildung 1: Publikationshäufigkeiten zu verschiedenen aktuellen Themen der Paar- und Familienpsychologie im Vergleich (PsycINFO)

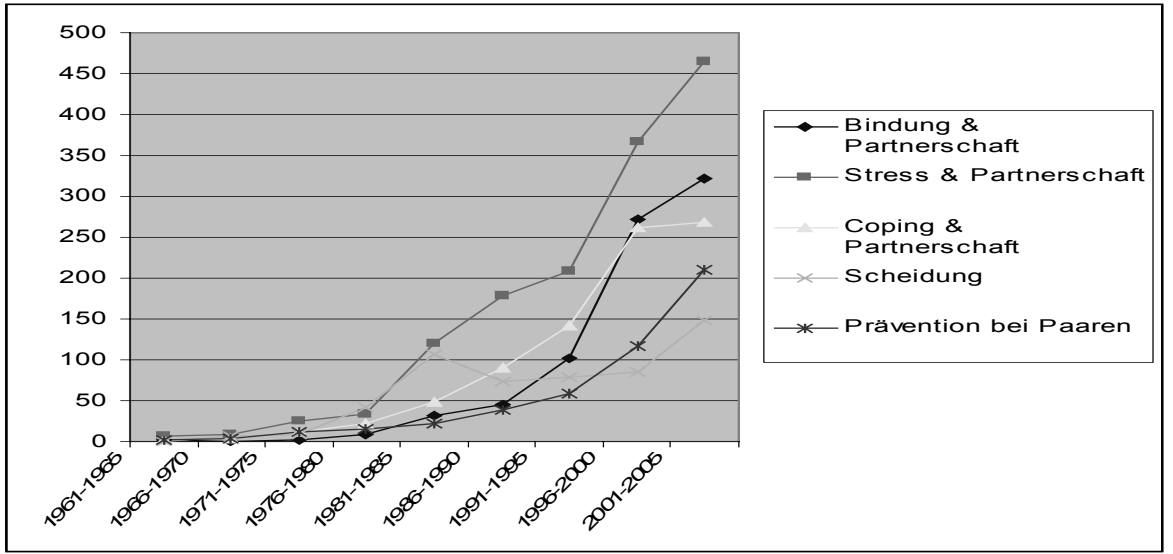

\section{Familienpsychologische Grundlagenforschung}

\section{Inhaltliche Schwerpunkte}

Es ist keine leichte Aufgabe, Bereiche innerhalb der Paar- und Familienpsychologie zu benennen, die aktuell die meiste theoretische Beachtung verdienen und die stärkste Forschungsaktivität stimulieren, da die paar- und familienpsychologischen Themen auch von verschiedenen Disziplinen innerhalb der Psychologie bearbeitet werden (z.B. Entwicklungspsychologie, Sozialpsychologie, Persönlichkeitspsychologie, Klinische Psychologie, Gesundheitspsychologie). Dennoch möchte ich den Versuch wagen, einige Trends nachzuzeichnen, welche in der Grundlagenforschung der Paarund Familienpsychologie der letzten Jahre einen nennenswerten Boom erfahren haben. Während in den 1970er und 1980er Jahren insbesondere die Interaktionsforschung zur Frage nach Unterschieden zwischen zufriedenen und unzufriedenen Paaren oder dysfunktionalen und funktionalen Familien im Vordergrund standen (Weiss/ Heyman 1997), erlebte Ende der 1980er Jahre bis Mitte der 1990er Jahre die Attributionsforschung insbesondere bei Paaren aber auch bei Familien ein gesteigertes Forschungsinteresse. Aktuell können neben einer Vielzahl von Forschungsthemen (wie z.B. die Bedeutung verschiedener Familienformen und ihre Lebensbedingungen, Familien in ökonomischer Deprivation und Armut, familiale Beziehungsmuster und ihre Entwicklung, Geschwister- und Zwillingsbeziehungen, Anpassung der Kinder an neue Familienformen und Scheidung, Sozialisation im Familienkontext und im sozialen Wandel, die Rolle der Väter in der Familie und für die Entwicklung der Kinder, Rollenteilung und moderne Paarbeziehungen, familiäre Gewalt, etc.) vier Schwerpunkte ausgemacht werden, die m.E. zurzeit die stärkste Resonanz im deut- 
schen Sprachraum aber auch international erfahren oder aber von ihrer Bedeutung her besondere Beachtung verdienen: (a) die Bindungsforschung bei Paaren und Familien, (b) die Scheidungsforschung, (c) die Stressforschung bei Paaren und Familien und (d) die Copingforschung bei Paaren und Familien. Alle vier Themen stimulierten in den letzten Jahren sowohl im deutschen Sprachraum wie auch international eine bemerkenswerte Forschungsaktivität, welche sich in einer Vielzahl von Publikationen niederschlug.

\section{Bindungsforschung bei Paaren}

Während in den Anfängen der Bindungsforschung die Mutter-Kind-Beziehungen im Vordergrund standen (Ainsworth/Bell/Stayton 1974; Bowlby 1969) hat die Bindungsforschung im Erwachsenenalter (vgl. wegweisende Beiträge von Bartholomew 1990; Gloger-Tippelt 2001; Hazan/Shaver 1987) und insbesondere in der Partnerschaft in den letzten Jahren einen unvergleichlichen Aufschwung erlebt, was sich in einer beeindruckenden und anhaltenden Häufigkeit an Publikationen zu diesen Fragestellungen in führenden Zeitschriften der Paar- und Familienpsychologie widerspiegelt, so etwa in den amerikanischen Fachzeitschriften Personal Relationships und Journal of Social and Personal Relationships. Ein Großteil dieser Publikationen bezieht sich dabei auf Bindung in Partnerschaften, davon ausgehend, dass das lebenslange Bedürfnis nach Nähe und Geborgenheit, welches man in der Kindheit durch die Bindung zu den Eltern (oder der Mutter) zu stillen versuchte, nun im Erwachsenenalter vom Partner zu erhalten strebt (vgl. Berlin/Cassidy 1999). Während einige interessante theoretische Beiträge z.B. zum Zusammenhang zwischen Bindung, Partnerschaftsentwicklung, Familienentwicklung und der Entwicklung der Kinder des Paares präsentiert wurden (z.B. Mikulincer/Florian/Cowan/Cowan 2002), stellen die meisten Beiträge empirische Arbeiten dar. Die Ergebnisse dieser Untersuchungen zeigen dabei relativ homogen, dass eine sichere Bindung mit einer höheren Partnerschaftsqualität und einem günstigeren Partnerschaftsverlauf (vgl. z.B. Banse 2004; Feeney 1999; Shaver/Hazan 1993) sowie einer besseren dyadischen Interaktion zwischen den Partnern einhergeht (z.B. Feeney 2002; Wampler/Shi/Nelson/Kimball 2003). Die Beziehung von sicher gebundenen Paaren ist dabei durch mehr Selbstöffnung, wechselseitiges Vertrauen, positive Emotionalität und Zärtlichkeit sowie eine günstigere Konflikt- und Problemlösung gekennzeichnet (z.B. Crowell/ Fraley/Shaver 1999; Guerrero 1998). Sicher gebundene Personen sind zudem häufiger verheiratet (rund $80 \%$ der verheirateten Paare weisen eine sichere Bindung auf) und führen meist einen gemeinsamen Haushalt mit dem Partner. Ängstlich-vermeidende, ängstlich-ambivalente und gleichgültig-vermeidende Paare, welche unsicher gebundene Paare repräsentieren, scheinen dagegen eine weniger tragfähige Partnerschaft aufzuweisen. Das Ausmaß an emotionaler Selbstöffnung ist bei diesen Bindungstypen reduziert, die Beziehungsdefinition häufig unklarer, die wechselseitige Aufeinanderbezogenheit schwächer ausgeprägt und die Stabilität der Beziehungen deutlich niedriger als diejenige von sicher gebundenen Paaren. Das höchste Risiko für Trennung/Scheidung weisen Paare mit einer gleichgültig-vermeidenden Frau und 
einem ängstlich-ambivalenten Mann auf, wohingegen Paare mit einer ängstlich-gebundenen Frau und einem vermeidend-gebundenen Mann durchaus längerfristig stabil sein können (vgl. Bierhoff/Grau 1999; Cina 1997; von Sydow /Ullmeyer 2000).

Neuere Studien fokussieren dabei nicht mehr nur diese direkten Assoziationen, sondern testen vermehrt auch Moderator- und Mediatormodelle unter Einbezug von Stress, Befinden, Bindung und Partnerschaftsqualität (vgl. Meyers/Landsberger 2002).

In neueren Beiträgen wird die Bindungstheorie auch im Rahmen der Emotionsregulation bei Paaren (z.B. im Zusammenhang mit der Regulation von Gefühlen wie Eifersucht, Hass, Angst vor Zurückweisung, Demütigung; vgl. Feeney 2005; Mikulincer/Shaver 2005) oder im Kontext der Copingforschung herangezogen. So zeigen beispielsweise Arbeiten von Lussier, Sabourin und Turgeon (1997) und Mikulincer und Florian (2001), dass Unterschiede im Bewältigungsverhalten der verschiedenen Bindungstypen beobachtbar sind. So praktizieren sicher gebundene Partner häufiger sachbezogenes Coping, ängstlich gebundene eher emotionsbezogenes und vermeidende Partner öfters vermeidendes Coping, wobei die Zuweisung nicht immer eindeutig möglich ist. Lussier et al. (1997) fanden zudem eine Moderatorwirkung des individuellen Copings im Zusammenhang mit dem Bindungsstil und der Partnerschaftsqualität. Insgesamt liegen jedoch erst wenige Studien zum Zusammenhang zwischen Stress, Coping und Partnerschaft (vgl. Feeney/Kirkpatrick 1995) respektive zu dyadischem Coping und Bindungstypen vor (Cina 1997).

Der Nutzen der Bindungsforschung für den klinischen Kontext (z.B. Paartherapie) wird in neueren Beiträgen ebenfalls reflektiert (z.B. Wampler et al. 2003), wonach eine Thematisierung von Bindungsaspekten in der Paartherapie in etlichen Fällen indiziert sein könnte.

\section{Bindungsforschung bei Familien}

Neben dem Aufschwung der Bindungsforschung bei Paaren konstatiert man erneut auch ein stärkeres Interesse an den Attachment-Theorien im Kontext der Familie allgemein. Dies wird beispielsweise durch die beiden im Jahr 2004 erschienenen Bücher von Liselotte Ahnert „Frühe Bindung: Entstehung und Entwicklung “ und Klaus und Karin Grossmann „Bindung: das Gefüge psychischer Sicherheit" dokumentiert. Diese beiden Bücher stehen für eine ganze Reihe von jüngst alleine im deutschen Sprachraum publizierten Schriften zur Bindungstheorie und ihrer Bedeutung beispielsweise im entwicklungspsychologischen oder klinischen Kontext (z.B. Spangler/Zimmermann 1995; Strauss/Buchheim /Kächele 2002). Das Thema der Bindung in der Familie hat dabei vor allem auch vor dem Hintergrund der wachsenden Mobilität, der weit verbreiteten Berufstätigkeit beider Eltern und der Fremdbetreuung von Kindern in Krippen und anderen vorschulischen Einrichtungen für Kinder (z.B. Kindertagesstätten) erneut an Aktualität und Brisanz gewonnen. Interessant ist jedoch auch die Frage, welchen Beitrag die Väter für den Bindungsaufbau ihrer Kinder leisten können und in Wirklichkeit in unserer Gesellschaft auch leisten.

Die Befunde der neueren Bindungsforschung, die auch hohen methodischen Ansprüchen genügen, sind für die Frage der Fremdbetreuung von Kindern in hohem 
Masse bedeutsam, wenn auch politisch häufig brisant. Entsprechend wichtig sind Schriften, wie die des NICHD Early Child Care Research Network (2003), welche der Frage nachgehen, welche Auswirkungen die Krippenbetreuung für das Befinden der Kinder hat. Die Studie zeigt, dass externalisierende Verhaltensprobleme der Kinder (z.B. aggressives Verhalten, Ungehorsam) und die Konflikthäufigkeit mit Erwachsenen (gemäß Angaben der Eltern, Erzieher und Lehrer) im Alter von viereinhalb Jahren respektive beim Eintritt in den Kindergarten signifikant positiv mit der Dauer der frühkindlichen familienexternen Kinderbetreuung korreliert sind - und dies weitgehend unabhängig vom Typ und von der Qualität der Krippenbetreuung. Ahnert und Lamb (2003) resümieren, dass Familien, die außerfamiliäre Kinderbetreuung in Anspruch nehmen, häufig ihr Betreuungsverhalten gegenüber ihren Kindern verändern, indem sie versuchen, die Bindung vor allem über eine affektive Interaktion zu den Zeiten, wo sie mit den Kindern zusammen sind (morgens und abends), aufrechtzuerhalten. Es zeigt sich jedoch, dass Eltern von tagesbetreuten Kindern häufig weniger responsiv und kontingent auf Stresssignale ihrer Kinder reagieren, was es schwierig machen kann, den emotionalen Bedürfnissen der Kinder gerecht zu werden. Hinzu kommt, dass lange Aufenthaltszeiten in außerfamiliärer Betreuung das Bindungsbedürfnis des Kindes erhöhen, die Eltern jedoch nicht adäquat darauf eingehen können, da sie nach einem langen Arbeitstag selber müde und erschöpft sind. Ahnert und Lamb (2003) rufen deshalb die Bedeutung der mütterlichen Sensitivität gegenüber den Bindungsbedürfnissen der Kinder gerade in Stresssituationen in Erinnerung und mahnen, dass die Auswirkungen von Fremdbetreuung durch diese Variable moderiert werden.

Dieser Forschungsstrang innerhalb der Familienpsychologie darf als von hoher gesellschaftlicher und familienpolitischer Bedeutung angesehen werden und es ist zu wünschen, dass in den nächsten Jahren eine Reihe weiterer relevanter Befunde zur Bindungsforschung publiziert werden.

\section{Scheidungsforschung bei Paaren und Familien}

Scheidungsursachen. Seit den 1990er Jahren hat sich die Paarforschung vermehrt mit der Frage von Scheidungsprädiktoren zu befassen begonnen. Dabei interessiert vor allem die Frage, welche Variablen bereits zu Beginn der Partnerschaft respektive zu einem möglichst frühen Zeitpunkt, den Verlauf der Paarbeziehung und deren Scheidungsrisiko vorhersagen lassen. Bahnbrechend in diesem Kontext waren vor allem die Arbeiten der Forschergruppe um John Gottman von der University of Washington in Seattle (z.B. Carrère/Buehlman/Gottman/Coan/Ruckstuhl 2000; Gottman 1994; Gottman/Coan/Carrère/Swanson 1998) sowie die Gruppe um Thomas Bradbury an der UCLA (z.B. Bradbury 1998; Bradbury/Fincham/Beach 2000; Karney/Bradbury 1995), welche eine Vielzahl von Veröffentlichungen zu dieser Fragestellung in den letzten Jahren vorgewiesen haben. Im deutschen Sprachraum wurden Längsschnittstudien von Bodenmann und Cina (2000), Bierhoff und Grau (1996), Brandtstätter und Felser (2003) sowie Schneewind et al. (1997) zu dieser Fragestellung publiziert. Aktuell läuft zudem eine mehrjährige prospektive Längsschnittstudie zur 
Frage, was Ehen zusammenhält, unter der Leitung von Klaus Schneewind und Eva Wunderer von der Universität München.

Wie mehrere Überblicksartikel zeigen (z.B. Bodenmann 2001; Karney/Bradbury 1995) liegt heute eine Reihe von gesichertem Wissen zu Risikofaktoren für Partnerschaften vor, welche eine richtige Vorhersage von Trennung und Scheidung aufgrund psychologischer Variablen im Durchschnitt mit mehr als 80\% Vorhersagegenauigkeit (richtige Zuordnung der Paare zur Gruppe der stabilen versus instabilen Paare) erlauben. Als wichtigste Prädiktoren für Scheidung erweisen sich dabei (a) Neurotizismus eines oder beider Partner und (b) Kompetenzdefizite der Partner bezüglich dyadischer Kommunikation (hohe Negativität bei gleichzeitig geringer Positivität in der Interaktion, negative Reziprozität bis hin zu eskalativen Verläufen und Zwangsprozess), Problemlösung (ineffiziente oder fehlende Problemlösung) und Stressbewältigung (dysfunktionale individuelle oder dyadische Stressbewältigung). Neben prospektiven Längsschnittstudien zeigen auch retrospektive Studien zu subjektiven Scheidungsgründen interessante Befunde (vgl. Schneider 1990). Analog zu den Längsschnittstudien führen auch die Geschiedenen selber (insbesondere die Frauen) die Scheidung häufig auf Kompetenzdefizite des Partners zurück, gefolgt von einem geringen Engagement für die Partnerschaft und emotionaler Entfremdung zwischen den Partnern, wie eine jüngst abgeschlossene deutsch-italienisch-schweizerische Studie zeigt (Bodenmann et al. 2005). Damit erhält die Aussage, dass insbesondere Kompetenzen der Partner zentral für das längerfristige Gelingen einer Partnerschaft sind weitere empirische Unterstützung. Entsprechend wichtig erweist sich die nach wie vor aktive Interaktionsforschung bei Paaren und Familien (vgl. Schneewind/Gerhard 2002), welche in neuerer Zeit neben ihrem Schwerpunkt auf der Positivität und Negativität in der dyadischen Interaktion (vgl. soziale Lerntheorien) vermehrt auch neue Aspekte fokussiert, wie supportives Verhalten oder dyadisches Coping (z.B. Bodenmann 2000; Cutrona 1996; Verhofstadt/Buysse/Ickes/De Clercq/Peene 2005), Annäherungs-Vermeidungs-Muster (z.B. Cauglin 2002) und Aggression und Gewalt in dyadischen Interaktionen (z.B. Lawrence/Bradbury 2001; Rogge/Bradbury 1999). Neben diesen empirischen Studien wurden auch neuere Modelle zum Verständnis von Scheidungen vorgeschlagen, so das sozial-physiologische Modell von Gottman (1994), das Vulnerabilitäts-Stress-Adaptationsmodell von Karney und Bradbury (1995) sowie im deutschen Sprachraum das Resilienz-Modell der Paarbeziehung von Lösel und Bender (1998) und das Stress-Scheidungs-Modell von Bodenmann (2004).

Scheidungsfolgen. Ein weiterer Strang der Scheidungsforschung beschäftigt sich mit den Folgen von Scheidung für die betroffenen Partner und die Kinder. Diese Fragestellung wird heute international meist in prospektiven Längsschnittstudien untersucht, welche ein relativ verlässliches Bild von den Folgen der Scheidung insbesondere für die Kinder geben (vgl. Amato 2001, 2003; Dreman 2000; Kelly 2000; Kelly/Emery 2003; Schmidt-Denter/Beelmann 1997; Schmitz/Schmidt-Denter 1999; Walper/Schwarz 1999). Dabei zeichnet sich ab, dass nicht nur die direkten und indirekten Auswirkungen der Scheidung für die weitere Entwicklung der Kinder und ihre Adaptation an das Ereignis relevant sind, sondern insbesondere die Qualität der Beziehung zwischen den Eltern respektive die Beziehung der Eltern zu ihren Kindern 
vor, während und nach der Scheidung (vgl. Cummings/Davies 2002; Krishnakumar/Buehler 2000; Morrison/Coiro 1999).

\section{Stressforschung bei Paaren und Familien}

Die Stressforschung bei Familien bildet seit den 1930er Jahren einen eigenständigen Strang innerhalb der Stressforschung, der allerdings lange von der Stressforschung bei Individuen kaum wahrgenommen wurde und sich völlig unabhängig von ihm entwickelte. Lange Zeit war das ABC-X-Modell (vgl. Hill 1949; McCubbin/Patterson 1983) das dominierende und prominenteste Stressmodell im Bereich der Familie, welches sich empirisch als äußerst fruchtbar erwies und eine Vielzahl von Studien stimulierte. Der eigentliche Aufschwung in der Stressforschung bei Paaren und Familien erfolgte allerdings erst zu Beginn der 1990er Jahre. Zeitgleich in den USA (z.B. Coyne/Smith 1991; DeLongis/O'Brien 1990) und in Europa (z.B. Bodenmann/Perrez 1991; Bodenmann 2000; Perrez 2000, 2005; Schneewind 1999) entwickelten Forscher unabhängig voneinander eigene Stress- und Coping-Modelle bei Paaren und Familien, die zunehmende Resonanz erhielten und die sich von der individuums-zentrierten Sicht von Stress und Coping (vgl. Lazarus/Folkman 1984) distanzierten. Dabei wurden Stresstaxonomien bei Paaren (z.B. Bodenmann 2000; Karney/Story/Bradbury 2005; Story/Bradbury 2004) und Familien (z.B. Perrez 2000; Schneewind 1999) vorgeschlagen, welche auch in neueren Forschungsarbeiten ihren Niederschlag gefunden haben. So dürfte sich v.a. die Unterscheidung und systematischere Auseinanderhaltung von paarinternem Stress (der innerhalb der Paarbeziehung entsteht, z.B. infolge von unterschiedlichen Zielen, Bedürfnissen, Konflikten oder auch der Sorge um den Partner) und paarexternem Stress (Stress, der außerhalb der Partnerschaft entsteht, d.h. primär nichts mit dem Partner zu tun hat) als nützlich erweisen, um Konfundierungen dieser beiden Stressarten (welche leider in einigen Forschungsarbeiten zu finden sind) zu vermeiden und damit die Reliabilität und Validität von Befunden zu Auswirkungen von Stress auf die Partnerschaft oder Familie zu erhöhen.

Wie erwähnt hat dabei die Stressforschung bei Paaren und Familien in den letzten Jahren einen regelrechten Aufschwung erfahren, der sich in einer wachsenden Zahl von Publikationen widerspiegelt (vgl. Bodenmann 1995, 2000, 2005; Cohan/Bradbury 1997; Karney et al. 2005; Revenson et al. 2005; Story/Bradbury 2004). Die meisten Arbeiten befassen sich dabei mit der Frage, welche Folgen (externer) Stress für das Paar- oder Familiensystem hat, wobei Effekte von Stress auf die dyadische oder familiäre Kommunikation (z.B. Bodenmann 2000, Neff/Karney 2004; Repetti 1989) und Affektregulation (z.B. Wilhelm/Perrez 2004, Wilhem/Schöbi/Perrez 2004), das psychische und physische Befinden der Partner und Familienmitglieder (z.B. Revenson et al. 2005), die Partnerschafts- oder Familienzufriedenheit (z.B. Bodenmann 2000) und das Scheidungsrisiko von Paaren (z.B. Bodenmann/Cina 2000; Rogge/Leonard/Bradbury 2005) untersucht werden. Die Studien zeigen dabei relativ homogen, dass vor allem Alltagsstress (,daily hassles“), nicht aber kritische Lebensereignisse signifikant negativ mit einer ungünstigeren Kommunikation (mehr 
Rückzug in der Kommunikation, mehr Hostilität und weniger Positivität), einem schlechteren Befinden der Partner, einer niedrigeren Partnerschaftsqualität und einem höheren Scheidungsrisiko einhergehen.

Repetti und Wood (1997) konnten zudem einen negativen Effekt von Stress auf das Erziehungsverhalten nachweisen.

Die hohe aktuelle Attraktivität von Stress und Partnerschaft respektive Stress und Familie als Forschungsthemen belegt auch das europäische Projekt zur Verträglichkeit von Beruf und Familie (,family life and professional work: Conflict and synergy"), welches die internen und externen Stressoren und Ressourcen von jungen Paaren mit Vorschulkindern im Kontext der Schnittstelle von Beruf und Familie untersucht. Das Projekt, welches in sieben Ländern (Belgien, Deutschland, Italien, Niederlande, Österreich, Portugal, Schweiz) läuft, kann dabei auf Daten (Selbstberichtdaten mittels Fragebögen und computerunterstützter ereignisnaher Protokollierung im Feld) einer beachtlichen Stichprobe von rund 1400 Paaren zurückgreifen.

Innerhalb der Stressforschung bei Paaren und Familien hat zudem die psychobiologische Forschung (physiologische und endokrinologische Parameter) in neuester Zeit zusehends an Bedeutung gewonnen. Inspiriert durch die frühen Arbeiten von Gottman und Levenson (1992) haben neuere Forschungsarbeiten zuverlässig nachweisen können, dass eine niedrige Partnerschaftsqualität mit schlechteren Immunfunktionen und einem niedrigeren physischen Befinden einher geht (vgl. KiecoltGlaser et al. 2003; Robles/Kiecolt-Glaser 2003). Partnerschaftskonflikte sind zudem nachweislich mit einem höheren Blutdruck und einer schnelleren Herzfrequenz assoziiert (vgl. Broadwell/Light 1999), wobei ein hostiler Interaktionsstil bei den negativ interagierenden Partnern mit einem höheren Katecholaminspiegel einher geht (vgl. Malarkey et al. 1994). Diese multi-methodale Erfassung des Zusammenspiels von psychischen, sozialen und biologischen Prozessen bei Paaren und Familien dürfte in nächster Zukunft weiter an Bedeutung gewinnen.

\section{Coping-Forschung bei Paaren und Familien}

Innerhalb der Stressforschung bei Paaren und Familien hat sich ein Konstrukt entwickelt, welches inzwischen einen breiten Niederschlag in der deutschsprachigen und amerikanischen Literatur gefunden hat, das dyadische Coping. Konzepte zur dyadischen (partnerschaftlichen) Stressbewältigung tauchten zeitgleich in den USA (,relationship-focused coping“ von Coyne/Smith 1991; „,empathic coping“ von DeLongis/O'Brien 1990) und im deutschen Sprachraum anfangs der 1990er Jahre auf (Bodenmann/Perrez 1991; Bodenmann 1995, 2000) und stimulierten seither international eine Vielzahl von theoretischen und empirischen Schriften, welche in den Büchern "Stress und Coping bei Paaren" von Bodenmann (2000), „Gemeinsame Stressbewältigung “ von Buchwald et al. (2003) oder dem Buch „Couples coping with stress: Emerging perspectives on dyadic coping " von Tracy Revenson, Karen Kayser und Guy Bodenmann im Jahr 2005 ihren fruchtbaren Niederschlag fanden. Der Begriff, der ursprünglich von Bodenmann eingeführt wurde und supportives, delegiertes oder gemeinsames Bewältigungsverhalten von Paaren umschrieb, wurde 
zudem in neuester Zeit auch auf Bewältigungsverhalten von anderen Dyaden (z.B. Freunden, Kollegen) ausgeweitet (vgl. Buchwald 2002), eine Entwicklung, der allerdings nicht unkritisch entgegengesehen wird, da dyadisches Coping (als CopingForm bei Paaren in Ergänzung zum individuellen Coping beider Partner einzeln) bewusst theoretisch und konzeptionell vom Begriff der sozialen Unterstützung (auch der sozialen Unterstützung in der Partnerschaft, vgl. Cutrona 1996) abgegrenzt wurde. Dies aus mehreren Gründen: (a) Unterstützung in der Partnerschaft ist qualitativ anders als Unterstützung durch paarexterne Personen, (b) Unterstützung durch den Partner ist nicht primär altruistisch motiviert, sondern dient dem unterstützenden Partner selber in hohem Maße, da es ihm nur so gut gehen kann, wie es dem anderen Partner geht; vgl. Interdependenz des Befindens in Paarbeziehungen und daher auch hohe Motivation beider Partner, den anderen in seiner Stressbewältigung zu unterstützen), (c) dyadisches Coping umfasst mehr als nur unterstützendes Verhalten, es beschreibt einen systemisch-prozessualen Akt des Sendens von Stresssignalen und des Eingehens auf diese Signale seitens des Partners, wobei neben dem unterstützenden (supportiven) dyadischen Coping auch das gemeinsame und delegierte dyadische Coping unterschieden werden, (d) dyadisches Coping kann auch negativer Ausprägung sein (hostiles, ambivalentes oder floskelhaftes dyadisches Coping) und kann in gewissen Situationen dysfunktional sein.

Eine Reihe von Studien unterstreicht die Bedeutung des dyadischen Copings im Kontext der Partnerschaft und Familie. So zeigt sich übereinstimmend, dass dyadisches Coping mit einem besseren dyadischen Funktionsniveau sowohl bei Selbstberichtdaten (z.B. Badr 2004; Bodenmann 1995, 2000; Walen/Lachman 2000) als auch bei Verhaltensbeobachtungsdaten (z.B. Bodenmann 2000; Cutrona/Suhr 1992; Pasch/ Bradbury 1998) einhergeht. Dyadisches Coping ist nicht nur deshalb mit einer höheren Partnerschaftszufriedenheit assoziiert weil es Spill-over-Prozesse von externem Stress des einen Partners auf die Gesamtdyade zu reduzieren hilft, sondern insbesondere weil dyadisches Coping das „Wir-Gefühl“ des Paares, das Gefühl wechselseitiger Unterstützung und damit Vertrauen, Intimität und Sicherheit fördert. Ferner belegt eine Reihe von Studien positive Zusammenhänge zwischen dyadischem Coping und dem Befinden der Partner respektive ihrer Adaptationsleistung an chronische Krankheiten (z.B. Acitelli/Badr 2005; Hagedoorn/Buunk/Kuijer/Wobbes/ Sanderman 2000; Manne et al. 2003; Revenson 1994; Rohrbaugh et al. 2002).

\section{Methodische Schwerpunkte}

In methodischer Hinsicht hat sich in den letzten Jahren ebenfalls viel getan. Obgleich gemäß Gottman und Notarius (2002) in der Paar- und Familiendiagnostik weiterhin international Fragebogenverfahren dominieren, obwohl die systematische Verhaltensbeobachtung in vielen Fällen die Methode der Wahl wäre, finden sich doch auch einige bemerkenswerte Neuerungen im Bereich der Diagnostik. So die Anwendung von impliziter Diagnostik im Rahmen der Paarforschung (Erfassung von Variablen, die nicht explizit zu erfassen vorgegeben werden oder die von den Probanden nicht 
willentlich kontrolliert werden können; vgl. Banse 2003), das Verfahren des VideoRecall (z.B. Welsh/Dickson 2005), endokrinologische und pyhsiologische Messungen bei Paaren und Familien (z.B. Fehm-Wolfsdorf/Groth/Kaiser/Hahlweg 1999; Gottman/Levenson 1992) sowie die computerunterstützte ereignisnahe Erfassung von Variablen im Feld (Perrez/Berger/Wilhelm 1998). Das von Perrez et al. (1998) vorgestellte Family Self Monitoring System (FASEM) (Mini-Palmtop-Computer) erlaubt die ereignisnahe Abbildung von verschiedenen Verhaltens- und Erlebensvariablen direkt im Feld und bei verschiedenen Protagonisten der Familie gleichzeitig. Die diagnostische Erfassung kann nach der Methode des Event-Sampling (sobald das Verhalten oder Erleben auftritt wird es protokolliert) oder des Time-Samplings (in vorgegebenen Zeitintervallen erinnert der Minicomputer einen daran, dass gewisse Fragen zu beantworten sind) erfolgen. Das System ist dabei so programmiert, dass es adaptiv in Abhängigkeit der beantworteten Items die weitere Fragenselektion vornimmt (z.B. Fragen zu sozialem Coping nur dann, wenn andere Personen anwesend sind), wobei das System eine adaptive Flexibilität aufweist. Innerhalb eines definierten Zeitraums (z.B. während einer Woche) können so mit hoher Reliabilität Variablen bei sämtlichen Familienmitgliedern erhoben werden. All diese genannten diagnostischen Neuerungen erlauben eine für viele Fragestellungen angemessenere Untersuchung dyadischer und familiärer Phänomene und tragen zu einer häufigeren Anwendung von multi-methodalen Ansätzen in der Paar- und Familiendiagnostik bei.

Mehr noch als im diagnostischen Bereich hat sich im Rahmen der Auswertung von Paar- und Familiendaten viel getan. Für dieses gestiegene Interesse an methodischen Fragestellungen und Auswertungsmethoden zeugt auch eine Spezialausgabe der Zeitschrift Journal of Family Psychology (Nr. 19, 2005) welche ihre Beiträge exklusiv diesem Thema widmete. Die hohe Komplexität von Paar- und Familiendaten (welche per definitionem abhängige Daten darstellen und meist interaktiven oder zirkulären Charakter haben) hat in den letzten Jahren immer mehr auch methodische Diskussionen stimuliert, wie solche Daten fachgerecht auszuwerten sind. Dabei wurde die Forderung laut, dass Verfahren verwendet werden sollten, welche der Spezifität dieser Variablen Rechnung tragen. Dies sind zum einen Multilevel-Analyseverfahren (vgl. HLM; Raudenbush/Brennan/Barnett 1995), zum anderen Verfahren, welche die Abhängigkeit der Daten kontrollieren (vgl. Cook 1998; Cook/Snyder 2005; Kashy/Kenny 2000; Ledermann/Bodenmann 2006; Neyer 1998). Obgleich in vielen Untersuchungen diese neueren Verfahren noch zu wenig Berücksichtigung finden, werden sie in einigen Jahren international zum Auswertungsstandard gehören.

\section{Familienpsychologische Anwendungsforschung}

Neben der familienpsychologischen Grundlagenforschung hat sich in den letzten Jahren ebenfalls sehr viel im Bereich der familienpsychologischen Anwendungsforschung getan. Den vielleicht größten Boom innerhalb der familienpsychologischen Anwendungsforschung hat in den letzten Jahren die Prävention von kindlichen Stö- 
rungen durch eine Verbesserung des elterlichen Erziehungsverhaltens oder der mütterlichen Responsivität erfahren.

\section{Präventionsangebote für Eltern}

Im Erziehungskontext wurden in den letzten Jahren mehrere Präventionsangebote entwickelt und wissenschaftlich evaluiert, welche von einzelnen Elternkursen über Hilfsmittel für Eltern (Ratgeber, CD-ROM) bis hin zu ganzen Präventionsprogrammen reichen. Im Zentrum stehen dabei meist Ansätze, welche den Eltern aufzeigen, wie sie angemessener mit schwierigen Erziehungssituationen umgehen und in $\mathrm{Ab}$ hängigkeit ihrer Ziele konsistent erziehen können. Während lange Zeit das Gordontraining im deutschen Sprachraum eine Vorrangstellung genossen hatte, wurde diese nun in den letzten Jahren durch neue Angebote aufgeweicht, so das Erziehungsangebot Starke Eltern - starke Kinder (Honkanen-Schoberth 2003), ein Programm, welches vor allem vom deutschen Kinderschutzbund stark gefördert wird, das aus den USA stammende STEP-Programm (Abidin 1996), welches in Deutschland von Klaus Hurrelmann an der Universität Bielefeld evaluiert wird oder das in Australien entwickelte und von Kurt Hahlweg in Deutschland aufgebaute Positive Parenting Program (Triple P) (z.B. Cina/Blattner-Bolliger/Bodenmann/Hahlweg/Sanders 2006; Sanders/Markie-Dadds/Tully/Bor 2000) oder im französischsprachigen Raum der von Jean-Pierre Pourtois entwickelte Elternkurs Education familiale (Pourtois/Desmet 2004). Diese Kursangebote für Eltern hat Klaus Schneewind zudem durch eine CD-ROM ,Freiheit in Grenzen “ für Eltern von Kindern im Alter von 612 Jahren und für Eltern von Jugendlichen erweitert. Ziel dieser CD-ROM ist es, den Eltern ein Erziehungskonzept aufzuzeigen, welches den Kindern die Entwicklung einer lebensbejahenden, selbständigen, leistungsbereiten und gemeinschaftsfähigen Persönlichkeit erlaubt. Als drei Hauptkomponenten günstigen elterlichen Verhaltens werden anhand von Videobeispielen (a) elterliche Wertschätzung für das Kind, (b) das elterliche Grenzen setzen sowie (c) das Gewähren von kindlicher Eigenständigkeit aufgezeigt.

Neben Kursen zur Förderung der Erziehungskompetenzen der Eltern, wurden auch weitere Angebote für Eltern oder werdende Eltern geschaffen. So hat Reichle (1999) ein strukturiertes, empirisch fundiertes Präventionsprogramm für werdende Eltern vorgestellt, welches als Bausteine (a) Einstellung auf Lebensveränderungen mit den damit einhergehenden Konsequenzen für das Zeitbudget und andere Aktivitäten, (b) gute Gesprächsführung (positive Reziprozität, Zuhörer- und Sprecherregeln), (c) Umgang mit belastenden Gefühlen (Entspannungstechniken, Ärgeranalyse, Ärgerkontrolle) sowie (d) Umgang mit Meinungsverschiedenheiten und Belastungen (konstruktive Konflikt- und Problemlösung) beinhaltet. Trainings zur Förderung der mütterlichen Sensitivität gegenüber den Kindern (vgl. weiter oben Ausführungen zur Bindungsforschung), die leider im deutschen Sprachraum noch weitgehend unbekannt sind und nur selten zur Anwendung gelangen, wurden in den letzten Jahren vor allem in den USA entwickelt und empirisch evaluiert (vgl. Dulmus/Rapp-Paglicci 2000; Bakermans-Kranenburg/van Ijzendoorn/Juffer 2003). 
Präventionsangebote für Paare und Familien

Neuere Studien belegen, dass Präventionsprogramme für Paare eine respektable Wirksamkeit aufweisen (vgl. Halford/Markman/Kline/Stanley 2003; Shadish/Baldwin 2003) und auch stärker im Selbstverständnis der Paar- und Familienpsychologie sowie in der Wahrnehmung der Bevölkerung verankert sind. Für eine verstärkte Wahrnehmung des Themas Prävention bei Paaren spricht auch die im Jahr 2004 gedruckte Sonderausgabe der Zeitschrift Family Relations sowie das 1999 erschienene Buch von Berger und Hannah „Preventive approaches in couple's therapy“, welches eine gute Übersicht über aktuelle Präventionsprogramme (v.a. in den USA) gibt.

Erfreulicherweise haben sich Präventionsangebote für Paare in den letzten Jahren, nachdem sie in den Vereinigten Staaten bereits seit den späten 70er Jahren ein fest verankertes Angebot darstellen, auch im deutschen Sprachraum durchzusetzen begonnen. So konnten mit dem EPL (Ein partnerschaftliches Lernprogramm) und seinen Weiterentwicklungen für Krisenpaare (KEK) sowie dem Freiburger Stresspräventionstraining für Paare (FSPT) wissenschaftlich fundierte Programme angeboten werden, welche in mehreren Studien ihre Wirksamkeit unter Beweis gestellt haben (vgl. Bodenmann/Shantinath 2004; Hahlweg/Markman/Thurmaier/Engl/Eckert 1998; Kaiser/Hahlweg/Fehm-Wolfsdorf/Groth 1998). Die beiden Programme haben zudem eine relative gute Breitenwirkung entfalten können, womit das Ziel einer universellen Prävention von Beziehungsstörungen weiter verfolgt werden kann. Diesem Ziel zuträglich sind auch die innovativen und kreativen Wege, welche Ragnar Beer (GeorgAugust Universität Göttingen) mit seiner Online-Paarberatung (www. theratalk.de) und Yves Hänggi (Universität Fribourg, $\mathrm{CH}$ ) mit seinem Internettraining für Eltern (www.elterntraining.ch) beschritten haben.

\section{Neuerungen in der Paartherapie}

Neben den genannten Präventionsangeboten finden sich auch in der Paartherapie Neuerungen, welche eine kurze Erwähnung verdienen (vgl. Tabelle 2). 
Tabelle 2: Klassische und neuere Komponenten in der verhaltenstherapeutischen Paartherapie

\begin{tabular}{lll}
\hline Methode & Inhalt & Ziel \\
\hline Reziprozitätstraining & $\begin{array}{l}\text { Realisieren und Umsetzen von } \\
\text { Positivität im Alltag, Verwöh- } \\
\text { nungstage }\end{array}$ & $\begin{array}{l}\text { Aufbau und Verstärkung von wechsel- } \\
\text { seitiger Positivität }\end{array}$ \\
Kommunikationstraining & $\begin{array}{l}\text { Kommunikationsregeln für den } \\
\text { Sprecher und den Zuhörer, struk- } \\
\text { turiertes Setting }\end{array}$ & $\begin{array}{l}\text { Verbesserung der dyadischen Kom- } \\
\text { munikation }\end{array}$ \\
Problemlösetraining & $\begin{array}{l}\text { 6-stufiges Schema zur Lösung von } \\
\text { Problemen }\end{array}$ & $\begin{array}{l}\text { Verbesserung der Lösung von Alltags- } \\
\text { problemen }\end{array}$ \\
3-Phasen-Methode & $\begin{array}{l}\text { Phase der Stressäußerung } \\
\text { (Trichtermethode), Phase der } \\
\text { dyadischen Unterstützung, Phase } \\
\text { des Feedbacks zu dieser Unter- } \\
\text { stützung }\end{array}$ & $\begin{array}{l}\text { Aufbau von Intimität durch Selbstöff- } \\
\text { nung, Erhöhung des dyadischen Co- } \\
\text { pings }\end{array}$ \\
\hline $\begin{array}{l}\text { Schaffung von mehr Verständnis } \\
\text { und Akzeptanz durch Distanzie- } \\
\text { rung vom Problem, eigene Frei- } \\
\text { räume, etc. }\end{array}$ & $\begin{array}{l}\text { Suche nach Kompromissen und Tole- } \\
\text { ranz in der Partnerschaft }\end{array}$ \\
\hline & &
\end{tabular}

So haben insbesondere Neil Jacobson und Andrew Christensen (UCLA) mit ihrer integrativen Paartherapie („Integrative behavioral couple therapy“) der Paartherapie neue Impulse gegeben. Aufbauend auf der Einsicht, dass nicht sämtliche störenden Bedingungen und Verhaltensweisen des Partners verändert werden können, schlagen die beiden Autoren einen Ansatz vor, welcher den Schwerpunkt auf die wechselseitige Akzeptanz legt. Dabei verstehen Jacobson und Christensen unter Akzeptieren nicht die Zustimmung zum Status Quo oder eine Form von Resignation, sondern die Fähigkeit eines Paares, die Probleme als Vehikel für mehr Intimität und Nähe zu sehen, indem das Problem gemeinsam (nicht gegeneinander) zu lösen versucht wird, wobei die Einsicht, dass Unterschiede zwischen den Partnern natürlicherweise vorliegen und auch durchaus bestehen können, ohne dass diese eine konstante Quelle von Konflikten sein müssen. Damit schlagen sie vor, den Versuch, den Partner nach seinen Vorstellungen verändern zu wollen, aufzugeben und stattdessen mehr Toleranz füreinander aufzubauen. Der Ansatz zielt somit vor allem auf die Förderung von wechselseitigem Verständnis und Empathie, die emotionale Distanzierung vom Problem (Entflechtung von Problem und Partnerschaft) sowie den Aufbau von mehr Selbständigkeit und Selbstpflege ab.

Eine zweite Neuerung hat Bodenmann mit der Bewältigungsorientierten Paartherapie vorgeschlagen, welche das Wissen aus der Stress- und Coping-Forschung bei Paaren im Kontext der Paartherapie konsequent umzusetzen versucht (Bodenmann 2004). Im Zentrum dieses Ansatzes steht die Förderung des dyadischen Copings bei Paaren durch die 3-Phasen-Methode, bei welcher die Partner lernen, sich externen Stress angemessen mitzuteilen (1. Phase), sich wechselseitig adäquat bei der Stressbewältigung zu unterstützen (2. Phase) und gemeinsam das dyadische Coping durch 
Feedback auszubauen (3. Phase). Beide Ansätze, sowohl der integrative Ansatz von Jacobson und Christensen wie auch der bewältigungsorientierte Ansatz finden in der paartherapeutischen Arbeit vermehrt Berücksichtigung.

Spannend sind in diesem Bereich auch neuere Analysen im Bereich der Wirksamkeitsforschung, wonach nicht nur Gruppenunterschiede zwischen der Interventionsund Kontrollgruppe nach den klassischen RCT-Designs (randomized control trials) analysiert werden, sondern in neueren Studien auch die Wirkmechanismen genauer unter die Lupe genommen werden. Die Studie von Schilling, Baucom, Burnett, Allen und Ragland (2003) gehört zu den ersten, welche solche Analysen vornahmen. Die Befunde beider Studien sind von hohem Interesse. So zeigten Schilling et al. (2003), in Übereinstimmung mit anderen Studien, dass kritisches Verhalten der Frau häufig für die Beziehungszufriedenheit günstiger ist als ihr positives Verhalten, ja, dass sogar eine Zunahme dieses kritischen Verhaltens nach der Paarintervention prospektiv günstig für die Verringerung des Rückfallrisikos ist. Diese Befunde bedürfen nun weiterer Replikation, könnten jedoch für die weitere Verbesserung von Interventionsmethoden von großer Bedeutung sein.

\section{Zusammenfassung und Ausblick}

In diesem Beitrag wurde versucht, insbesondere auf Neuerungen und aktuelle Strömungen in der Paar- und Familienpsychologie einzugehen, wobei ich mir bewusst bin, dass dabei auch viele wichtige Forschungslinien und -projekte nicht berücksichtigt wurden, welche für die Paar- und Familienpsychologie von großer Relevanz sind. Es ging daher auch nicht darum, einen repräsentativen Überblick über all das zu geben, was aktuell läuft, sondern einige wichtige Linien herauszugreifen, die besondere theoretische und empirische Aktivitäten in der Paar- und Familienpsychologie stimuliert haben. Dabei wurde deutlich, dass die Paar- und Familienpsychologie als neue Disziplin eine sehr ermutigende Entwicklung erfahren hat und in den letzten Jahren zu einer vollwertigen Disziplin innerhalb der psychologischen Grundlagenund Anwendungsfelder herangereift ist. Dies schlägt sich nicht nur in den wissenschaftlichen Aktivitäten nieder, sondern auch in der institutionellen Verankerung, welche sich in der Gründung von Familienforschungsinstituten und Fachverbänden oder Breitbandveröffentlichungen (z.B. Online-Familienhandbuch) widerspiegelt.

Trotz dieser erfolgreichen Geschichte der Paar- und Familienpsychologie im deutschen Sprachraum und international bleiben auch Wünsche an eine weitere Entwicklung offen. Diese beziehen sich neben den bereits angesprochenen methodischen Anforderungen bei der Auswertung von Paar- und Familiendaten insbesondere auf Forschungsthemen oder praktische Anwendungsbereiche, die noch mehr Resonanz verdienen. So darf man getrost hoffen, dass Themen wie Armut und Gewalt in der Familie (vgl. Forschungsarbeiten von Sabine Walper in München zu diesen Themen), die Frage der Transmission von Familienwerten und Beziehungsmustern in Abhängigkeit verschiedener Familienformen oder insgesamt familiäre Sozialisationsbedingungen (z.B. Arbeiten von Manfred Hofer in Mannheim, Peter Noack in 
Jena und Hans-Georg Voss in Darmstadt) noch eine Reihe von Studien stimulieren. Auch Themen wie binationale Partnerschaften und Familien oder ein interkultureller Vergleich von Familien (vgl. hierzu Forschungsarbeiten von Hans-Georg Voss) sowie die Rolle der Väter (vgl. hierzu Forschungsarbeiten von Wassilios Fthenakis) sind bisher aus psychologischer Perspektive marginal untersucht worden. Andere Themen wie die Evolutionspsychologie, Attraktionsforschung und austauschtheoretische Arbeiten sind seit Längerem forschungsrelevant, haben jedoch in den letzten Jahren kein gesteigertes Interesse verzeichnet. Dagegen stellt man im Anwendungsbereich erstaunlicherweise eine große Lücke bei sekundären Präventionsangeboten für Geschiedene fest. Während es Präventionsprogramme für Kinder, die von der Scheidung ihrer Eltern betroffen sind, gibt (vgl. Fthenakis et al. 1995), fehlen bisher weitgehend wissenschaftlich fundierte Programme für Geschiedene. Bedenkt man die hohen Scheidungsraten in westlichen Industrieländern und die Tatsache, dass Folgeehen in der Regel eine noch höhere Scheidungswahrscheinlichkeit aufweisen, wird deutlich wie wichtig solche Initiativen sein könnten. Die Liste möglicher und wünschenswerter Forschungsthemen, welche in der Paar- und Familienforschung hohe Aufmerksamkeit erfahren, könnte noch beliebig weitergeführt werden (z.B. Attraktionsforschung, Gewalt und Familie, Armut und Familie, Evolutionspsychologie, austauschtheoretische Arbeiten). Dies soll hier nun allerdings nicht getan werden, erstens um nicht die Tatsache zu schmälern, dass bereits viel in der Paar- und Familienforschung läuft und zweitens im Wissen, dass diese Themen in der Zukunft weitere Impulse erfahren werden. So dynamisch und fruchtbar die Paar- und Familienpsychologie in der kurzen Zeit ihres Bestehens gestartet ist, so lebendig und kreativ wird sie sich auch weiterentwickeln, dies nur schon deshalb, weil ihr Forschungsgegenstand von höchster gesellschaftlicher, wissenschaftlicher und politischer Bedeutung ist. Es bleibt zu wünschen und hoffen, dass die Politikerinnen und Politiker in den verschiedenen Ländern den reichen Fundus an wissenschaftlichen Erkenntnissen der Paar- und Familienforschung zur Kenntnis nehmen und ihren Entscheidungen in der Familienpolitik zugrunde legen.

\section{Literatur}

Abidin, R. R. (1996). Early childhood parenting skills: Program manual. Odessa, FL: Psychological Assessment Resources.

Acitelli, L. K./Badr, H. J. (2005). My illness or our illness? Attending to the relationship when one partner is ill. In: Revenson, T. A./Kayser, K./Bodenmann, G. (eds.). Couples coping with stress. Emerging perspectives on dyadic coping (pp. 121-136). Washington, DC: American Psychological Association.

Ahnert, L. (Hrsg.). (2004). Frühe Bindung. Entstehung und Entwicklung. München: Reinhardt.

Ahnert, L./Lamb, M. E. (2003). Shared care: Establishing a balance between home and child care. Child Development, 74, p. 1044-1049.

Ainsworth, M. D. S./Bell, S. M./Stayton, D. J. (1974). Infant-mother attachment and social development: Socialization as a product of reciprocal responsiveness to signals. In: Richards, M. P. M. (Ed.). The integration of a child into a social world (pp. 99-135). London: Cambridge University Press. 
Amato, P. R. (2001). Children of divorce in the 1990s: An update of the Amato and Keith (1991) meta-analysis. Journal of Family Psychology, 15(3), p. 355-370

Amato, P. R. (2003). Reconciling divergent perspectives: Judith Wallerstein, quantitative family research, and children of divorce. Family Relations, 52, p. 332-339;

Badr, H. (2004). Coping in marital dyads: A contextual perspective on the role of gender and health. Personal Relationships, 11, p. 197-211.

Bakermans-Kranenburg, M. J./van Ijzendoorn, M. H./Juffer, F. (2003). Less is more: Metaanalyses of sensitivity and attachment interventions in early childhood. Psychological Bulletin, 129, p. 195-215.

Banse, R. (2004). Attachment style and marital satisfaction: Evidence for dyadic configuration effects. Journal of Social and Personal Relationships, 21, p. 273-282.

Banse, R. (2003). Partnerschaftsdiagnostik. In: Grau, I./Bierhoff, H. W. (Hrsg.). Sozialpsychologie der Partnerschaft (S. 13-42). Göttingen: Hogrefe.

Bartholomew, K. (1990). Avoidance of intimacy: An attachment perspective. Journal of Social and Personal Relationships, 7, p. 147-178.

Berger, R./Hannah, M. T. (Eds.) (1999). Preventive approaches in couples' therapy. Philadelphia: Brunner/Mazel.

Berlin, L. R./Cassidy, J. (1999). Relations among relationships: Contributions from attachment theory and research. In: Cassidy, J. (Ed.). Handbook of attachment: Theory, research, and clinical applications (pp. 688-712). New York: The Guilford Press.

Bierhoff, H. W./Grau, I. (1996). Zur Vorhersage der Trennung in romantischen Beziehungen. Zeitschrift für Differentielle und Diagnostische Psychologie, 17, S. 251-261.

Bierhoff, H. W./Grau, I. (1999). Romantische Beziehungen. Bindung, Liebe, Partnerschaft. Göttingen: Hans Huber.

Bodenmann, G. (2000). Stress und Coping bei Paaren. Göttingen: Hogrefe.

Bodenmann, G. (2001). Risikofaktoren für Scheidung: Ein Überblick. Psychologische Rundschau, 52, S. 85-95.

Bodenmann, G. (2004). Verhaltenstherapie mit Paaren. Bern: Huber.

Bodenmann, G. (2005). Dyadic coping and its significance for marital functioning. In: Revenson,T./Kayser, K./Bodenmann, G. (Eds.). Couples coping with stress: Emerging perspectives on dyadic coping (pp. 33-50). Washington, DC: American Psychological Association.

Bodenmann, G./Charvoz, L./Bradbury, T. N./Bertoni, A./Iafrate, R./Giuliani, G./Bahnse, R./Behling, J. (2005, revised). The role of stress in divorce: A retrospective study in three nations. Journal of Personal and Social Relationships.

Bodenmann, G./Cina, A. (2000). Stress und Coping als Prädiktoren für Scheidung: Eine prospektive Fünf-Jahres-Längsschnittstudie. Zeitschrift für Familienforschung, 12, S. 5-20.

Bodenmann, G./Perrez, M. (1991). Dyadisches Coping - eine systemische Betrachtungsweise der Belastungsbewältigung in Partnerschaften. Zeitschrift für Familienforschung, 3, S. 425.

Bodenmann, G./Shantinath, S. D. (2004). The Couples Coping Enhancement Training (CCET): A new approach to prevention of marital distress based upon stress and coping. Family Relations, 53 (5), p. 477-484.

Bowlby, J. (1969). Attachment and loss. London: Hogarth Press.

Broadwell, S. D./Light, K. C. (1999). Family support and cardiovascular responses in married couples during conflict and other interactions. International Journal of Behavioral Medicine, 6, p. 40-63.

Bradbury, T. N. (1998). The developmental course of marital dysfunction. Cambridge. The Cambridge University Press. 
Bradbury, T. N./Fincham, F. D./Beach, S. R. H. (2000). Research on the nature and determinants of marital satisfaction: A decade review. Journal of Marriage and the Family, 62, p. 964-980.

Brandtstädter, J./Felser, G. (2003). Entwicklung in Partnerschaften. Risiken und Ressourcen. Bern: Huber.

Buchwald, P. (2002). Dyadisches Coping in mündlichen Prüfungen. Göttingen: Hogrefe.

Buchwald, P./Schwarzer, C./Hobfoll, S. E. (2003). Stress gemeinsam bewältigen. Göttingen: Hogrefe.

Carrère, S./Buehlman, K. T./Gottman, J. M./Coan, J. A./Ruckstuhl, L. (2000). Predicting marital stability and divorce in newlywed couples. Journal of Family Psychology, 14, p. 42-58.

Cauglin, J. P. (2002). The demand/withdraw pattern of communication as a predictor of marital satisfaction over time: Unresolved issues and future directions. Human Communication Research, 28, p. 49-85.

Cina, A. (1997). Dyadisches Coping bei verschiedenen Bindungstypen. Unveröffentlichte Lizentiatsarbeit. Fribourg: Institut für Familienforschung und -beratung der Universität Fribourg.

Cina, A./Blattner-Bolliger, D./Bodenmann, G./Hahlweg, K./Sanders, M. (2006). Triple P: (Positive Parenting Program): Theoretischer und empirischer Hintergrund und erste Erfahrungen im deutschsprachigen Raum. Zeitschrift für Familienforschung, 1-2006, S. 66-88.

Cohan, C. L./Bradbury, T. N. (1997). Negative life events, marital interaction, and the longitudinal course of newlywed marriage. Journal of Personality and Social Psychology, 73, p. 114-128.

Cook, W. L. (1998). Integrating models of interdependence with treatment evaluation in marital therapy research. Journal of Family Psychology, 12, p. 529-542.

Cook, W. L./Snyder, D. K. (2005). Analyzing nonindependent outcomes in couple therapy using the Actor-Partner Interdependence Model. Journal of Family Psychology, 19, p. 133-141.

Coyne, J. C./Smith, D. A. F. (1991). Couples coping with a myocardial infarction: A contextual perspective on wives' distress. Journal of Personality and Social Psychology, 61, p. 404-412.

Crowell, J. A./Fraley, R. C./Shaver, P. R. (1999). Measurement of individual differences in adolescent and adult attachment. In: Cassidy, J./Shaver, P. R. (Eds.). Handbook of attachment: Theory, research, and clinical applications (pp. 434-465). New York: Guilford Press.

Cutrona, C. E. (1996). Social support in couples. Marriage as a resource in times of stress. Thousand Oaks, CA: Sage.

Cutrona, C. E./Suhr, J. A. (1992). Controllability of stressful events and satisfaction with spouse supportive behavior. Communication Research, 19, p. 154-174.

Dehle, C./Larsen, D./Landers, J. E. (2001). Social support in marriage. The American Journal of Family Therapy, 29, p. 307-324.

Cummings, E. M./Davies, P. T. (2002). Effects of marital conflict on children: Recent advances and emerging themes in process-oriented research. Journal of Child Psychology and Psychiatry, 43, p. 31-63.

DeLongis, A./O'Brien, T. (1990). An interpersonal framework for stress and coping: An application to the families of alzheimer's patients. In: Stephens, M. A. P./Crowther, J. H./Hobfoll, S. E./Tennenbaum, D. L. (Eds.). Stress and coping in later-life families (pp. 221-240). New York: Hemisphere Publishing Corporation.

Dreman (2000). The influence of divorce on children. Journal of Divorce and Remarriage, 32, p. 41-71.

Dulmus, C. N./Rapp-Paglicci, L. A. (2000). Prevention of mental disorders in children and adolescents: Future research. Families in Society: The Journal of Contemporary Human Services, 81, p. 294-303.

Feeney, J. A. (1999). Adult romantic attachment and couple relationships. In: Cassidy, J./Shaver, P. R. (Eds.). The handbook of attachment: Theory, research, and clinical applications (pp. 353-377). New York: Guilford Press. 
Feeney, J. A. (2002). Attachment, marital interaction, and relationship satisfaction: A diary study. Personal Relationships, 9, p. 39-55.

Feeney, J. A. (2005). Hurt feelings in couple relationships: Exploring the role of attachment and perceptions of personal injury. Personal Relationships, 12, p. 253-271.

Feeney, J. A./Kirkpatrick, L. A. (1995). Effects of adult attachment and presence of romantic partners on physiological responses to stress. Journal of Personality and Social Psychology, 70 , p. $255-270$.

Fehm-Wolfsdorf, G./Groth, T./Kaiser, A./Hahlweg, K. (1999). Cortisol response to marital conflict depend on marital interaction quality. International Journal of Behavioral Medicine, 6, p. 207-227.

Fthenakis, W. E./Textor, M. R. (2001). Online-Familienhandbuch. www.familienhandbuch.de.

Fthenakis, W. E./Chow, S./Gemar, K. (1995). Gruppeninterventionsprogramm für Kinder mit getrennt lebenden oder geschiedenen Eltern. Herausgeber: LBS-Initiative Junge Familie. Weinheim: Beltz.

Gloger-Tippelt, G. (2001). Bindung im Erwachsenenalter. Bern: Huber.

Gottman, J. M. (1994). What predicts divorce? Hillsdale, NJ: Erlbaum.

Gottman, J. M./Coan, J./Carrère, S./Swanson, C. (1998). Predicting marital happiness and stability from newlywed interactions. Journal of Marriage and the Family, 60, p. 5-22.

Gottman, J. M./Levenson, R. W. (1992). Marital process predictive of later dissolution: Behavior, physiology, and health. Journal of Personality and Social Psychology, 63, p. 221-233.

Gottman, J. M./Notarius, C. I. (2002). Marital research in the 20th century and a research agenda for the 21 th century. Family Process, 41, p. 159-197.

Guerrero, L. K. (1998). Attachment-style differences in the experience and expression of romatic jealousy. Personal Relationships, 5, p. 273-291.

Hagedoorn, M./Buunk, B. P./Kuijer, R. G./Wobbes, T./Sanderman, R. (2000). Couples dealing with cancer: Role and gender differences regarding psychological distress and quality of life. Psycho-Oncology, 9, p. 232-242.

Hahlweg, K./Markman, H. J./Thurmaier, F./Engl, J./Eckert, V. (1998). Prevention of marital distress: Results of a German prospective-longitudinal study. Journal of Family Psychology, 12, p. 543-556.

Halford, W. K./Markman, H. J./Kline, G. H./Stanley, S. M. (2003). Best practice in couple relationship education. Journal of Marital and Family Therapy, 29(3), p. 385-406.

Hazan, C./Shaver, P. (1987). Romantic love conceptualized as an attachment problem. Journal of Personality and Social Psychology, 52, p. 511-524.

Hill, R. (1949). Families under stress. New York: Harper.

Honkanen-Schoberth, P. (2003). Starke Kinder brauchen starke Eltern. Der Elternkurs des Deutschen Kinderschutzbundes. München: Urania.

Jacobson, N. S./Christensen, A. (1996). Integrative behavioral couple therapy. New York: Norton.

Kaiser A./Hahlweg K./Fehm-Wolfsdorf G./Groth T. (1998) The efficacy of a compact psychoeducational group training program for married couples. Journal of Consulting and Clinical Psychology 66, p. 753-760.

Karney, B. R./Bradbury, T. N. (1995). The longitudinal course of marital quality and stability: A review of theory, method, and research. Psychological Bulletin, 118, p. 3-34.

Karney, B. R./Story, L. B./Bradbury, T. N. (2005). Marriages in context: Interactions between chronic and acute stress among newlyweds. In: Revenson, T. A./Kayser, K./ Bodenmann, G. (Eds.). Emerging perspectives on couples' coping with stress. Washington, DC: APA.

Kashy, D. A./Kenny, D. A. (2000). The analysis of data from dyads and groups. In: Reis, H./Judd, C. M. (Eds.). Handbook of research methods in social psychology (pp. 451-477). New York: Cambridge University Press. 
Kelly, J. B. (2000). Children's adjustment in conflicted marriage and divorce: A decade of review of research. Journal of the American Academy of Child and Adolescent Psychiatry, 39, p. 963-973.

Kelly, J. B./Emery, R. E. (2003). Children's adjustment following divorce: Risk and resilience perspective. Family Relations, 52, p. 352-362

Kiecolt-Glaser, J. K./Bane, C./Glaser, R./Malarkey, W. B. (2003). Love, marriage, and divorce: Newlyweds' stress hormones foreshadow relationship changes. Journal of Consulting and Clinical Psychology, 71, p. 176-188.

Krishnakumar, A./Buehler, C. (2000). Interparental conflict and parenting behaviors. A metaanalytic review. Family Relations, 49(1), p. 25-44.

Lawrence, E./Bradbury, T. N. (2001). Physical aggression and marital dysfunction: A longitudinal analysis. Journal of Family Psychology, 15, p. 135-154.

Lazarus, R. S./Folkman, S. (1984). Stress, appraisal, and coping. New York: Springer.

Ledermann, T./Bodenmann, G. (2006). Moderator- und Mediatoreffekte bei dyadischen Daten: Zwei Erweiterungen des Akteur-Partner-Interdependenz-Modells. Zeitschrift für Sozialpsychologie, 37, S. 27-40.

Lösel, F./Bender, D. (1998). Risiko- und Schutzfaktoren in der Entwicklung zufriedener und stabiler Ehen: Eine integrative Perspektive. In: Hahlweg, K./Baucom, D. H./Bastine, D. H./ Markman, J. H. (Hrsg.). Prävention von Trennung und Scheidung - internationale Ansätze zur Prädiktion und Prävention von Beziehungsstörungen (S. 27-66). Stuttgart: Kohlhammer.

Lussier, Y./Sabourin, S./Turgeon, C. (1997). Coping strategies as moderators of the relationship between attachment and marital adjustment. Journal of Social and Personal Relationships, 14, p. 777-791.

Malarkey, W./Kiecolt-Glaser, J. K./Pearl, D./Glaser, R. (1994). Hostile behavior during marital conflict alters pituitary and adrenal hormones. Psychosomatic Medicine, 56, p. 41-51.

Manne, S./Ostroff, J./Sherman, M./Glassman, M./Ross, S./Goldstein, L./Fox, K. (2003). Buffering effects of family and friend support on associations between partner unsupportive behaviors and coping among women with breast cancer. Journal of Social and Personal Relationships, 20, p. 771-792.

McCubbin, H. I./Patterson, J. M. (1983). The family stress process: The double ABCX model of adjustment and adaptation. Marriage and Family Review, 6, p. 7-37.

Meyers, S. A./Landsberger, S. A. (2002). Direct and indirect pathways between adult attachment style and marital satisfaction. Personal Relationships, 9, p. 159-172.

Mikulincer, M./Florian, V. (2001). Attachment style and affect regulation - implications for coping with stress and mental health. In: Fletcher, G./Clark, M. (Eds.). Blackwell handbook of social psychology: Interpersonal processes (pp. 537-557). Oxford; UK: Blackwell Publishers.

Mikulincer, M./Florian, V./Cowan, P. A./Cowan, C. P. (2002). Attachment security in couple relationships: A systemic model and its implications for family dynamics. Family Process, 41, p. 405-434.

Mikulincer, M./Shaver, P. R. (2005). Attachment theory and emotions in close relationships: Exploring the attachment-related dynamics of emotional reactions to relational events. Personal Relationships, 12, p. 149-168.

Morrison, D. R./Coiro, M. J. (1999). Parental conflict and marital disruption: Do children benefit when high-conflict marriages are dissolved? Journal of Marriage and the Family, 61, p. 626-637.

National Institute of Child Health and Human Development Early Child Care Research Network. (2003). Does amount of time spent in child care predict socio-emotional adjustment during the transition to kindergarten? Child Development, 74, p. 976-1005. 
Neff, L. A./Karney, B. R. (2004). How does context affect intimate relationships? Linking external stress and cognitive processes within marriage. Personality and Social Psychology Bulletin, 30, p. 134-148.

Neyer, F. J. (1998). Zum Umgang mit dyadischen Daten: Neue Methoden für die Sozialpsychologie. Zeitschrift für Sozialpsychologie, 29, S. 291-306.

Pasch, L. A./Bradbury, T. N. (1998). Social support, conflict, and the development of marital dysfunction. Journal of Consulting and Clinical Psychology, 66, p. 219-230.

Perrez, M. (2000). Psychologie des Familien- und Paarstresses: Forschungsentwicklungen. In: Schneewind, K. A. (Hrsg.). Familienpsychologie im Aufwind. Brückenschlag zwischen Forschung und Praxis (S. 69-88). Göttingen: Hogrefe.

Perrez, M. (2005). Störungen im Kindes- und Jugendalter - Grundlagen und Störungen im Entwicklungsverlauf. In: Schlottke, P. F./Silbereisen, R. K./Schneider, S./Lauth,G. W. (Hrsg.). Enzyklopädie der Psychologie (Band 5; S. 193-246). Göttingen: Hogrefe.

Perrez, M./Berger, R./Wilhelm, P. (1998). Die Erfassung von Belastungserleben und Belastungsverarbeitung in der Familie: Self-Monitoring als neuer Ansatz. Psychologie in Erziehung und Unterricht, 45, S. 19-35.

Pourtois, J.-P./Desmet, H. (2004). L'Education implicite. Paris: P.U.F.

Raudenbush, S. W./Brennan, R. T./Barnett, R. C. (1995). A multivariate hierarchical model for studying psychological change within married couples. Journal of Family Psychology, 9, p. 161-174.

Reichle, B. (1999). Wir werden Eltern. Ein Kurs zur Vorbereitung auf die erste Elternschaft. Weinheim: Juventa.

Repetti, R. L. (1989). Effects of daily workload on subsequent behavior during marital interaction: The roles of social withdrawal and spouse support. Journal of Personality and Social Psychology, 57, p. 651-659.

Repetti, R. L./Wood, J. (1997). Effects of daily stress at work on mother's interactions with preschoolers. Journal of Family Psychology, 11, p. 90-108.

Revenson, T. A. (1994). Social support and marital coping with chronic illness. Annals of Behavioural Medicine, 16, p. 122-130.

Revenson, T./Kayser, K./Bodenmann, G. (2005). Couples coping with stress: Emerging perspectives on dyadic coping. Washington, DC: American Psychological Association.

Robles, T. F./Kiecolt-Glaser, J. K. (2003). The physiology of marriage: Pathways to health. Physiology \& Behavior, 79, p. 409-416.

Rogge, R. D./Bradbury, T. N. (1999). Till violence does us part: The differing roles of communication and aggression in predicting adverse marital outcomes. Journal of Consulting and Clinical Psychology, 67, p. 340-351.

Rogge, R. D., Leonard/Bradbury, T. N. (2005). Prediction of marital outcomes in Caucasian and African-American couples. Manuscript in preparation.

Rohrbaugh, M. J./Cranford, J. A./Shoham, V./Nicklas, J. M./Sonnega, J. S./Coyne, J. C. (2002). Couples coping with congestive heart failure: Role and gender differences in psychological distress. Journal of Family Psychology, 16, p. 3-13.

Sanders, M. R./Markie-Dadds, C./Tully, L. A./Bor, W. (2000). The Triple P-positive parenting program: A comparison of enhanced, standard, and self-directed behavioral family intervention for parents of children with early onset conduct problems. Journal of Consulting and Clinical Psychology, 68, p. 624-640.

Schilling, E. A./Baucom, D. H./Burnett, C. K./Allen, E. A./Ragland, L. (2003). Altering the course of marriage: The effect of PREP communication skills acquisition on couples' risk of becoming maritally distressed. Journal of Family Psychology, 17(1), p. 41-53. 
Schmidt-Denter, U./Beelmann, W. (1997). Kindliche Symptombelastungen in der Zeit nach einer ehelichen Trennung - eine differentielle und längsschnittliche Betrachtung. Zeitschrift für Entwicklungspsychologie und Pädagogische Psychologie, 29(1), S. 26-42.

Schmitz, H./Schmidt-Denter, U. (1999). Die Nachscheidungsfamilie sechs Jahre nach der elterlichen Trennung. Zeitschrift für Familienforschung, 3, S. 28-55.

Schneewind, K. A. (1991). Familienpsychologie. Stuttgart: Kohlhammer.

Schneewind, K. A. (1999). Familienpsychologie (2. Auflage). Stuttgart: Kohlhammer.

Schneewind, K. A. (Hrsg.) (2000). Familienpsychologie im Aufwind. Brückenschlag zwischen Forschung und Praxis. Göttingen: Hogrefe.

Schneewind, K. A./Gerhard, A.-K. (2002). Relationship personality, conflict resolution, and marital satisfaction in the first five years of marriage. Family Relations, 51, p. 63-71.

Schneewind, K./Vascovics, L. A./Buba, H./Knopp, V./Rost, H./Schlehlein, B./Sierwald, W./Weiß, J. (1997). Optionen der Lebensgestaltung junger Ehen und Kinderwunsch. Stuttgart: Kohlhammer.

Schneider, N. F. (1990). Woran scheitern Partnerschaften? Subjektive Trennungsgründe und Belastungsfaktoren bei Ehepaaren und nichtehelichen Lebensgemeinschaften. Zeitschrift für Soziologie, 19, S. 458-470.

Shadish, W. R./Baldwin, S. A. (2003). Meta-analysis of MFT interventions. Journal of Marriage and Family Therapy, 29, p. 547-579.

Shaver, P. R./Hazan, C. (1993). Adult romantic attachment: Theory and evidence. In: Perlman, D./Jones, W. (Eds.). Advances in personal relationships (Vol. 4, pp. 29-70). London: Jessica Kingsley.

Spangler, G./Zimmermann, P. (Hrsg.) (1995). Die Bindungstheorie. Grundlagen, Forschung und Anwendung. Stuttgart: Klett Cotta.

Story, L. B./Bradbury, T. N. (2004). Understanding marriage and stress: Essential questions and challenges. Clinical Psychology Review, 23, p. 1139-1162.

Strauß, B./Buchheim, A./Kächele, H. (2002). Klinische Bindungsforschung. Stuttgart: Schattauer.

Verhofstadt, L. L./Buysse, A./Ickes, W./De Clercq, A./Peene, O. J. (2005). Conflict and support interactions in marriage: An analysis of couple's interactive behavior and on-line cognition. Personal Relationships, 12, p. 23-42.

von Sydow, K./Ullmeyer, M. (2000). Paarbeziehung und Bindung. Eine Meta-Inhaltsanalyse von 63 Studien, publiziert zwischen 1987 und 1997. Psychotherapie, Psychosomatik und medizinische Psychologie, 50, p. 1-15.

Walen, H. R./Lachman, M. E. (2000). Social support and strain from partner, family, and friends: Costs and benefits for men and women in adulthood. Journal of Social and Personal Relationships, 17, p. 5-30.

Walper, S./Pekrun, R. (Hrsg.) (2001). Familie und Entwicklung. Perspektiven der Familienpsychologie. Göttingen: Hogrefe.

Walper, S./Schwarz, B. (1999). Was wird aus den Kindern? Chancen und Risiken für die Entwicklung von Kindern aus Trennungs- und Scheidungsfamilien. Weinheim: Juventa.

Wampler, K. S./Shi, L./Nelson, B. S./Kimball, T. G. (2003). The adult attachment interview and observed couple interaction: Implications for an intergenerational perspective on couple therapy. Family Process, 42, p. 497-515.

Weiss, R. L./Heyman, R. E. (1997). A clinical overview of couples interactions. In: Halford, W. K./Markman, H. J. (Eds.). Clinical handbook of marriage and couples interventions (pp. 13-41). New York: Wiley \& Sons.

Welsh, D. P./Dickson, J. W. (2005). Video-recall procedures for examining subjective understanding in observational data. Journal of Family Psychology, 19, p. 62-71. 
Wilhelm, P./Perrez, M. (2004). How is my partner feeling in different daily-life settings? Accuracy of spouses' judgments about their partner's feelings at work and at home. Social Indicators Research, 67, p. 183-246.

Wilhem, P./Schöbi, D./Perrez, M. (2004). Frequency estimates of emotions in everyday life from a diary method's perspective: A comment on Scherer et al.'s survey-study "emotions in everyday life". Social Science Information, 43, p. 647-665.

Eingereicht am: 19.06.2005

Akzeptiert am: 27.07.2005

\section{Anschrift des Autors}

Prof. Dr. Guy Bodenmann

Universität Fribourg

Institut für Familienforschung und -beratung

Rue Faucigny 2

CH-1700 Fribourg

Email: Joseguy.bodenmann@unifr.ch 